\title{
Multitemporal SAR RGB Processing for Sentinel-1 GRD Products: Methodology and Applications
}

\author{
Donato Amitrano, Raffaella Guida, Member, IEEE, Giuseppe Ruello, Member, IEEE
}

\begin{abstract}
The Sentinel-1 mission has finally reached its maturity with the launch of the second Sentinel radar. Among the products delivered by the agency, the ground range detected class is raising more and more interest among users due to its reduced computational demand for information extraction and availability on cloud exploitation platforms, like the Google Earth Engine. In this work, we present a novel multitemporal processing chain suitable to be applied to Sentinel-1 ground range detected products to obtain RGB images using a series of single polarization detected images. These products aim at being the equivalent for the recently introduced Level-1 $\alpha$, exploiting a texture measure instead of the interferometric coherence, to properly render and enhance the presence of built-up areas. The discussion is supported by experiments showing the reliability of this newly introduced class of products in classic synthetic aperture radar applications like image photo-interpretation, flood mapping, and long term urban area monitoring.
\end{abstract}

Index Terms-multitemporal synthetic aperture radar, sentinel-1, image enhancement, classification, flood mapping, urban areas, archive data

\section{INTRODUCTION}

Starting from 2014, the launch of the Copernicus Programme is determining an unprecedented availability of satellite data [1] acquired at different wavelengths. This fact is particularly new for the SAR community which, for the first time, is experiencing an abundance of data going beyond the most consolidated practices developed in the literature for information extraction and, most of all, the visualization of time series data. Research is now paying an increasing attention to this topic [2]-[7] which is crucial for the expansion of the user community, thus favoring the development of the satellite data downstream market.

Among the products delivered by the radar Sentinel, there is a category that is gaining more and more interest in the user community, i.e. that of ground range detected (GRD) products. An obvious reason for that can be found in the cumbersome computational load behind any processing of complex Sentinel-1 data when adequate hardware architectures and storage units are not available. It should be sufficient that basic preprocessing of a time-series of six complex images (calibration, deburst, coregistration, and standard despeckling) using the snap platform takes approximately 11 hours on a high-end 8-cores, 128 GB RAM machine, and accounts for more than $120 \mathrm{~GB}$ storage. GRD products, instead, are

Donato Amitrano and Raffaella Guida are with the Surrey Space Centre, University of Surrey, Guildford, UK. email: \{d.amitrano, r.guida\}@surrey.ac.uk

Giuseppe Ruello is with the Department of Electrical Engineering and Information Technology, University of Napoli Federico II, Napoli, Italy. email: ruello@unina.it pre-processed images made available for download by the European Space Agency (ESA) via the Sentinels Data Hub. This allows users to handle more friendly products, both from the viewpoint of the memory consumption and that of the preprocessing to be applied before the dataset can be input to some information extraction algorithm [8]. In this case, the pre-processing of the equivalent time-series of six GRD products accounts for about 2.5 hours and $30 \mathrm{~GB}$ storage. The increasing interest around this class of products is also due to their availability on platforms allowing for massive cloud processing (such as the Google Earth Engine [9]-[11]).

In this context, being able to provide remote sensing data users with value-added products facilitating any following analysis is becoming the real game-changing factor in the downstream market sector.

The use of RGB compositions to visualise time-series information and to help the detection of land cover changes has been already exploited in the literature. Leaving aside the polarimetry world, in which color composition of multipolarization channels is more straightforoward and well consolidated in the literature [7], [12], [13], the general trend of the literature on this topic is to exploit temporal color composites to enhance the occurrence of certain phenomena. As an example, [5], [14], used RGB composites to highlight and extract flooded areas. Fires were addressed in [15]. Reference [16] used this technique to assess building damages as a consequence of a typhoon. Alves et al. [4] exploited threetemporal color composites for enhancing the visualization of Titan drainage networks using Cassini data.

Other works addressed the topic more generally, introducing frameworks for the generation of general-purposes temporal composites for improving data visualisation and the understanding of nonexpert remote sensing users. Recently, Schmitt et al. [17] proposed a new technique for color transferring from optical to SAR images which seems to be promising for visualisation purposes. Reference [3] showed how to combine temporal indicators in a single RGB frame representative of the dynamics of the imaged scene. Reference [2], exploited change detection principles to introduce a user-friendly RGB composition (known as Level-1 $\alpha$, hereinafter L-1 $\alpha$ ) allowing to easily detect changes in the imaged scene. In particular, the authors suggested to exploit two intensity channels (reference and test images) and their interferometric coherence to generate a balanced, user-friendly color image, in which the association color-feature respects, for some classes, the natural color palette, thus enhancing the user experience with data [18]. Clearly, the exploitation of the interferometric coherence requires a complex data input. However, as anticipated, complex 
Sentinel-1 data are very demanding in terms of processing time and memory, which might make the uptake of such processing chain difficult if either a good remote sensing background or high performance architectures are not available. These problems can be overcome by exploiting GRD products, that are more user friendly, application-oriented (see as an example [8], [19]), and less demanding from the viewpoint of the hardware necessary to handle them.

The objective of this paper is to provide a new processing chain leading to the definition of a new class of products (suitable for multisensor data fusion through access to archive images) carrying the same semantics of (complex) L- $1 \alpha$ without the necessity to use the phase information. This is achieved with the introduction of a texture measure in the multitemporal processing chain. The introduced novelties can be summarized as follows: i) introduction of a new class of products named as Detected Level-1 $\alpha$ (hereinafter DL-1 $\alpha$ ); ii) exploitation of texture as an information source for the generation of multitemporal color composites; iii) application of the principles of multitemporal data fusion to multisource data.

The paper is organized as follows. In Section II the proposed processing chain is discussed in detail. Semantic differences between complex and detected L- $1 \alpha$ images are discussed in Section III Applications, with special focus to image photo-interpretation, flood mapping, and urban area monitoring, are discussed in Section IV Conclusions are drawn at the end of the work.

\section{Methodology}

The proposed processing chain is largely inspired by that illustrated in [2], leading to the definition of change-detection oriented color composite (starting from complex data) in which the phase information is exploited to enhance the presence of urban areas. The main objective is now to introduce another class of products able to convey the same information using incoherent data as input.

To this end, the workflow depicted in Fig. 11 has been conceived. It starts from detected images, which are coregistered and radiometrically calibrated to compensate orbit and sensor effects. Despeckling is applied for enhancing the images information content. The use of temporal filters, such as the De Grandi filter [20], allowing to reduce speckle without degrading the spatial resolution or altering the texture significantly, is suggested. Cross-calibration and data quantization are implemented using the variable amplitude level equalization (VALE) method presented in [2]. In such way, unchanged scene objects exhibit the same reflectivity value in all the images of the time series.

As explained in [2], the selection of the time-series reference image (i.e. the one against which changes are evaluated) should be application-oriented (see Section IV for details). Following the schema reported in Fig. 11 this image is associated with the blue band of the output RGB composition. The test image (i.e. the one in which changes are measured) is associated with the green band. A texture measure able to convey on the urban area an information comparable to that provided by the interferometric coherence used in [2] is exploited as red band. To this end the data range is introduced:

$$
d_{r}=\max W-\min W, \quad d_{r} \in[0,255],
$$

where $W$ is the matrix given by a window sliding all over the re-quantized image. The data range is basically an edge detector. It is expected to be low in homogeneous areas and high in heterogeneous ones.

An example of the behavior of the data range texture is provided in Fig. 2. The imaged scene (acquired by the Sentinel-1 constellation on 5 January 2017) concerns the city of Chennai (India) with its surroundings. In particular, the lower row of the picture concerns a homogeneous agricultural area as seen, respectively, on the temporal-filtered SAR image (Fig. 2a) and on the data range map calculated in a moving window of 5 (Fig. 2b), 9 (Fig. 2c), and 13 pixels (Fig. 2d). The upper row of the picture is relevant to a a high texture urban area taken, respectively, from the SAR image Fig. 2e and from the corresponding data range map calculated for a moving window of 5 (Fig. 2f), 9 (Fig. 2g), and 13 pixels (Fig. 2h).

As aforementioned, the data range is low where the scene is homogeneous. Therefore, in agricultural areas, the effect is the enhancement of borders between crops. Fields with higher vegetation present typically higher values of texture, and consequently exhibit a slightly higher data range if compared with bare soils. As general effect, the more the window size increases, the more the image will be blurred, and fine details like man-made targets will suffer from.

In the urban area, the data range has its maximum response due to the alternation of strong and weak scatterers (namely, buildings and shadows). In this scenario, the effect of the variation of the texture window size is more evident. The 5pixel window size does not affect significantly the resolution and preserves the information content conveyed by the texture band. An increase of the sliding window dimension tends to broaden the data range over high texture areas. This is due to the higher likelihood of having more bright and dark pixels in the same window which alters the scene semantics in the 13pixels window size case (see Fig. 2h). As a general guideline, a dimension of 5 or 7 pixel well fits the resolution of the products here discussed.

Once the three bands of the composition have been defined, we can merge them into the final RGB map. As shown in the workflow of Fig. 11 (see the lower branch of the diagram), images acquired by multiple sensors can be merged into a unique RGB frame. The dashed line in the graphics indicates an optional flow which can be employed while dealing with specific applications, like long term urban area monitoring, as detailed in Section IV-C In this case, the processing chain is doubled, in the sense that the datacubes corresponding to the two sensors are processed separately following the first part of the diagram. The fusion is implemented after the VALE cross-calibration. In fact, as detailed in [2], this processing block transforms the normalized radar return from a distributed target (or backscatter coefficient, or sigma nought) into digital numbers proportional to it according to the time series metrics 


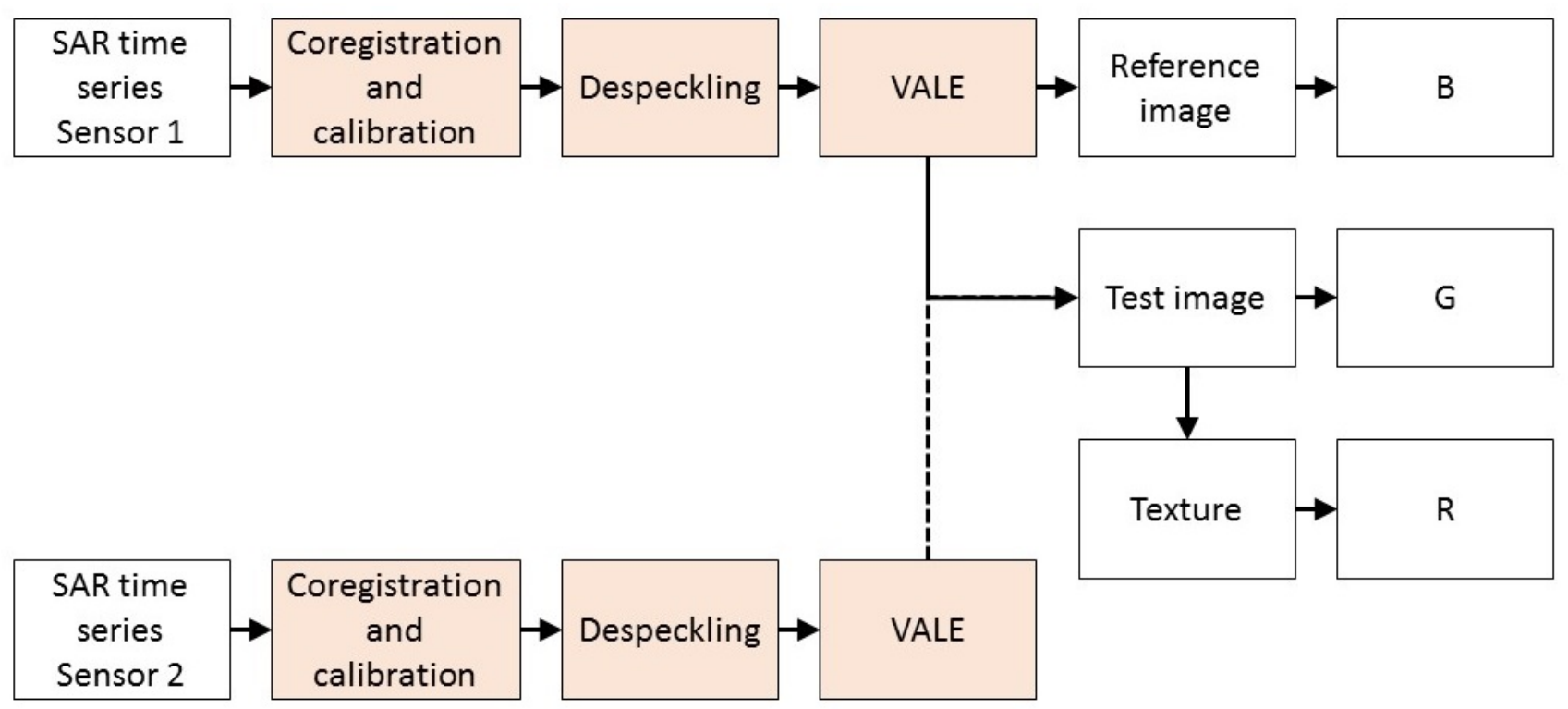

Fig. 1: Workflow for the generation of Level- $1 \alpha$ products starting from detected images. Optionally, data acquired by different SAR sensors can be exploited in the processing of the RGB composition (see lower branch of the diagram). Blocks with filled background indicate processes, those with white one stand for products.

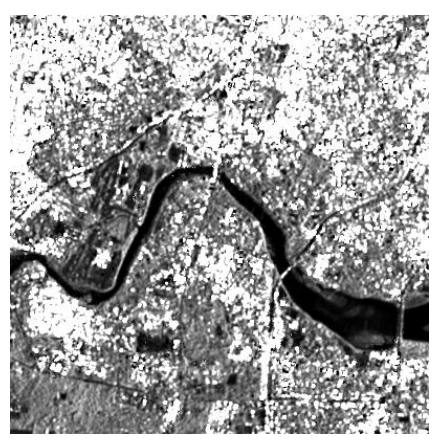

(a)

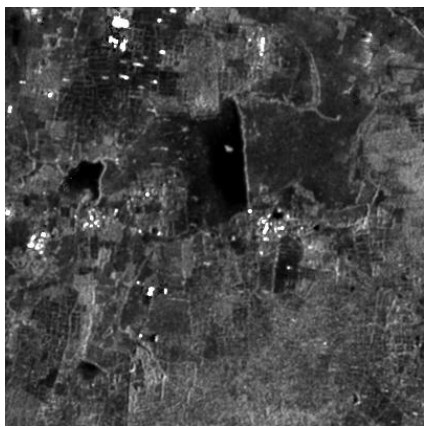

(e)

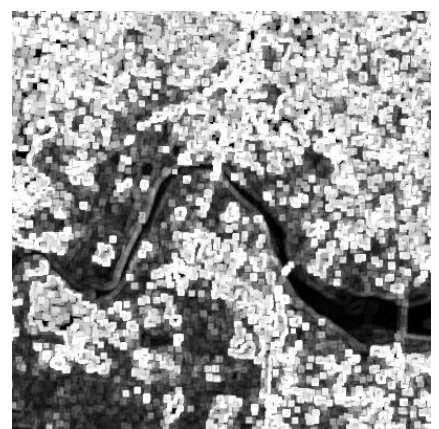

(b)

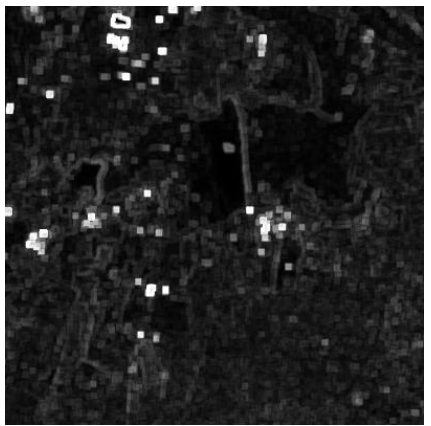

(f)

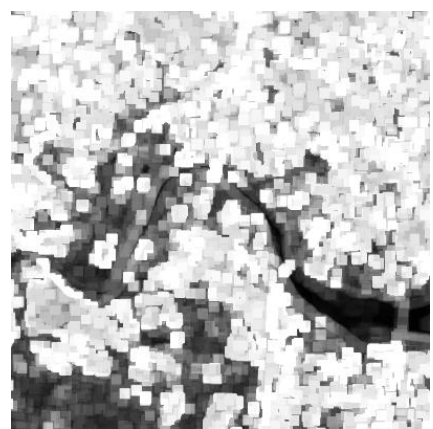

(c)

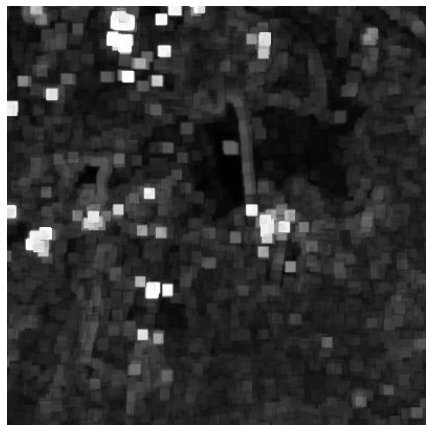

(g)

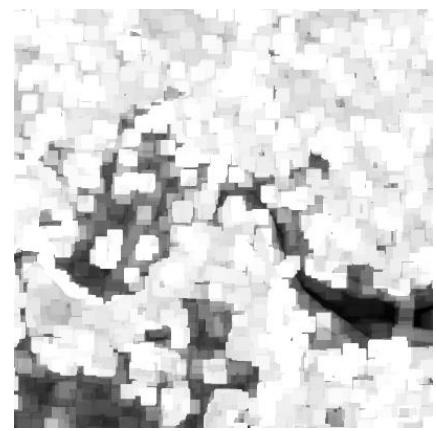

(d)

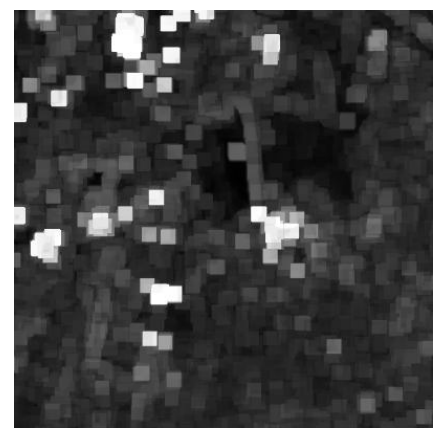

(h)

Fig. 2: Data range texture behavior as a function of the land cover and of the window dimension. Upper row: high texture urban area as rendered on (a) the SAR detected image and the data range texture map calculated in a (b) $5 \times 5$, (c) $9 \times 9$, and (d) $13 \times 13$ window. Lower row: low texture agricultural area as rendered on (e) the SAR detected image and the data range texture map calculated in a (f) $5 \times 5$, (g) $9 \times 9$, and (h) $13 \times 13$ window.

calculated using the VALE algorithm. These digital numbers, whatever is the SAR sensor acquiring the original images, are spatially, geometrically and radiometrically feature-consistent and this makes possible their pixel-based fusion into the final

RGB frame. 


\section{SEMANTIC COMPARISON WITH LEVEL- $1 \alpha$ PRODUCTS}

Before moving to applications,some examples assessing the differences between the L- $1 \alpha$ products based on using the interferometric coherence and the DL- $1 \alpha$ products exploiting the texture information are reported. In general, it is good practice to perform an assessment of the two classes of products on urban areas, thus highlighting the differences between the information from the interferometric coherence and that from the data range texture.

To this end, two RGB compositions generated as indicated in [2] (i.e. with the interferometric coherence associated with the red band) and as discussed above (i.e. with the data range texture associated with the red band, see Fig. 1) are considered. The study area concerns the city of Chennai (India). The reference image (blue band) and the test image (green band) have been acquired on 5 January 2017 and 11 April 2017, respectively.

In Fig. 3a a low density urban area as seen on standard $\mathrm{L}-1 \alpha$ products is shown. The same area as seen on the DL- $1 \alpha$ products is depicted in Fig. $3 \mathrm{~b}$. The reader should note that, in this case, no significant semantic differences arise between the two compositions. This is due to the fact that when the highly reflective scatterer (i.e. the building) is quite isolated, the pixel value dynamics in the SAR image is high when the operating window encloses building pixels as well as background pixels (belonging to the soil or shadow class). Therefore, the building will be rendered in white (thanks to the high reflectivity contribution of the two amplitude channels), while its edge is enhanced and displayed in red. This effect is identical to that of the coherence [2]. However, the reader should note that the DL- $1 \alpha$ product appears more blurred in correspondence of built-up pixels. This is due to the fact that when the data range is calculated in proximity of isolated buildings and the window centered on bare soil or shadow areas, its value is still high due to the difference in the reflectivity between built-up and background pixels.

When a high density urban area is considered, the interferometric coherence is expected to be high all over the patch, due to the dominance of phase-stable built-up pixels with respect to background pixels. Therefore, built-up pixels are still rendered in white, except for areas of phase-stability loss due to orbit incongruence or not-optimal choice of the coherence window dimension or shadowing effects or presence of stochastic scatterers in the calculation window [6], [21], [22]. Conversely, when the data range texture is used, in presence of continuous urban texture, it can happen, especially working at Sentinel-1 medium resolution, that no significant background return is found in the texture window. This makes the data range low and, as consequence, the built-up feature to appear in cyan, as only the intensity channels are contributing. This situation is depicted in Fig. 3c and Fig. 3d, in which a high density urban area is represented as appears in a standard $\mathrm{L}-1 \alpha$ product and in the DL- $1 \alpha$ product, respectively. In this case, the reader should easily note that the highest density urban area is rendered differently on the two products (white on the left, cyan on the right) due to the different behavior of the interferometric coherence and of the data range texture on

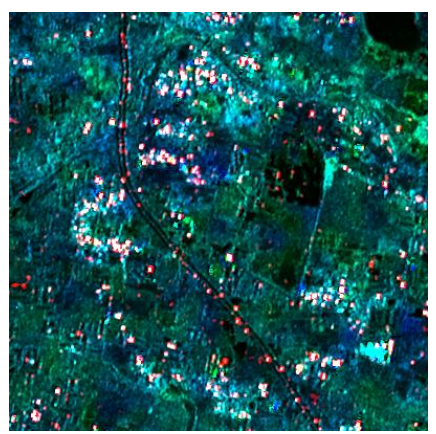

(a)

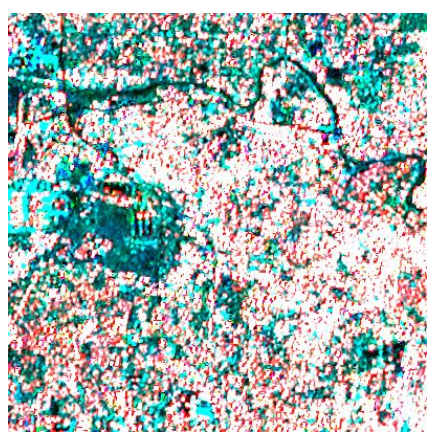

(c)

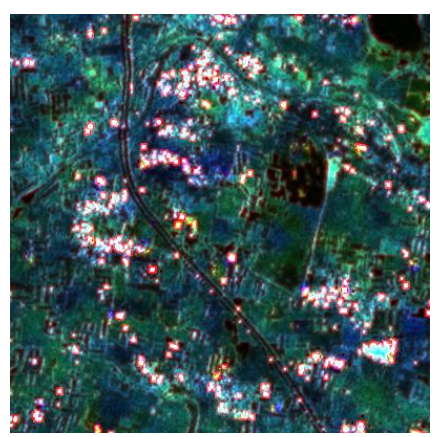

(b)

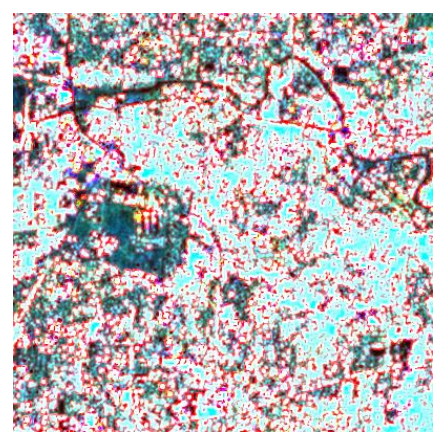

(d)
Fig. 3: Chennai, India. Semantic comparison between the L1$\alpha$ products proposed in [2] and the DL- $1 \alpha$ products here discussed. A low density urban area as seen on (a) L- $1 \alpha$ products and (b) DL- $1 \alpha$ products. A high density urban area as seen on (c) L- $1 \alpha$ products and (d) DL- $1 \alpha$ products.

this image feature.

Summarizing, the proposed DL- $1 \alpha$ products represent a quick solution for building change-detection RGB composites allowing to easily detect changes in the imaged scene. Main differences with respect to already available L- $1 \alpha$ images, obtained starting from complex data and exploiting the phase signal information, concern the rendering of the built-up area. For this feature, the performance of the detected products is slightly lower since the usage of the texture information causes blurring and a more fragmented semantics. On the other hand, detected products can be obtained with a much lower computational load. Their use is advised when high computational power is not available, as well as when the application does not require the highest detail on the built-up feature. This is the case of continuous monitoring of urban areas at regional scale, as detailed in Section IV-C

\section{Applications}

\section{A. Photo-interpretation}

In this Section, the user interpretability enhancement provided by the proposed DL- $1 \alpha$ products is quantitatively analyzed through a perceptive experiment. Thirty individuals with different expertise in remote sensing were asked to observe different representations of satellite data. They voted for the class they think was the dominant one in the patch under analysis choosing among "Urban area", "River", "Lake", 


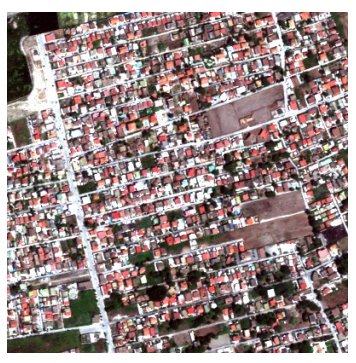

(a)

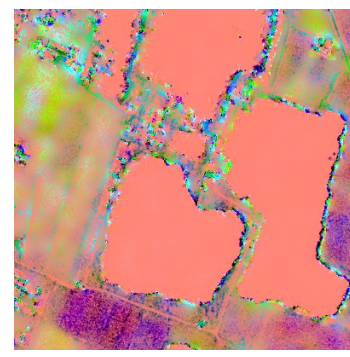

(b)

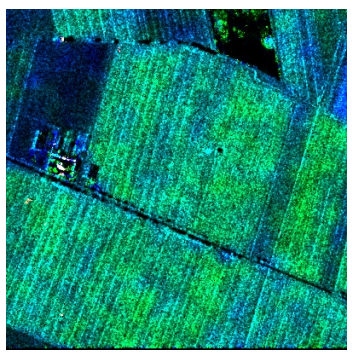

(c)

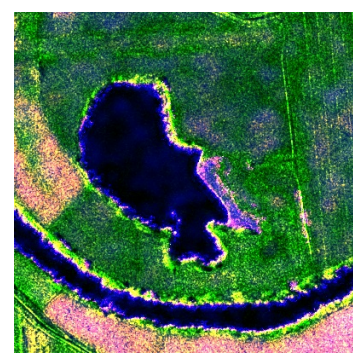

(d)

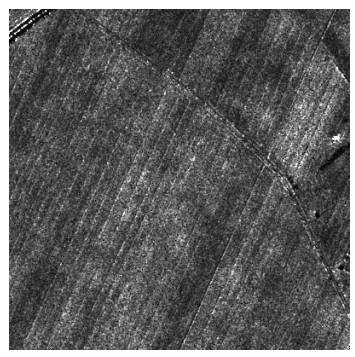

(e)

Fig. 4: Examples of the images used in the perceptive experiment. (a) Natural color optical image, (b) PCA, (c) DL-1 $\alpha$ product, (d) Level-1 $\beta$ product, and (e) standard SLC SAR image treated with multitemporal despeckling.

TABLE I: Results of the perceptive test. Values reported in the table refer to the percentage of successfull interpretation per class and per product type.

\begin{tabular}{ccccccccc}
\hline \hline Product type & Urban & River & Lake & Vegetation & Crops & Bare soil & Sea & Overall \\
\hline \hline DL-1 $\alpha$ & 74.3 & 100 & 69.2 & 33.3 & 76.2 & 40.0 & 84.6 & 62.2 \\
SLC & 78.9 & 100 & 58.3 & 26.9 & 80.0 & 17.1 & 66.6 & 52.5 \\
Natural color & 100 & 100 & 61.9 & 41.9 & 74.0 & 71.4 & 80.0 & 72.6 \\
Level-1 $\beta$ & 84.4 & 100 & 85.7 & 45.6 & 73.1 & 10.0 & 95.8 & 70.0 \\
PCA & 90.0 & 90.9 & 75.0 & 22.8 & 66.6 & 19.5 & 66.6 & 54.7 \\
\hline \hline
\end{tabular}

"Vegetation", "Crops", "Bare soil", "Sea". Each person voted for 20 scnes randomly selected from a database of 100 patches containing optical natural color images, principal component representations (PCA), DL- $1 \alpha$ products, Level- $1 \beta$ products [3], and standard SLC SAR images treated with multitemporal despeckling [20]. Examples of the images used in the test are provided in Fig. 4.

The results of the test are provided in TABLE I The best interpretation performance is registered for the features in which the shape or the texture is dominant, as in the case of the "River" and "Urban" classes. The most confusing classes among those included in the test were "Vegetation" and "Bare soil". In these cases, the interpretation capability of non-expert users was hampered by the lack of characteristics recalling standard representations of such objects when represented at microwave wavelength. An exception was the "Vegetation" class in Level- $1 \beta$ products, for which the success score is comparable to that of natural color images. Probably, this is due to the fact that this feature is displayed in green in such composition [3], thus helping an effective linkage with real world appearance of that class

Overall, as expected, the best interpretation performance is that of natural color satellite images, with a pecentage of correct interpretation higher than $70 \%$. Level- $1 \beta$ products also performed quite well, with an overall interpetation success of $70 \%$. The DL- $1 \alpha$ products here proposed had an overall success percentage of $62.2 \%$, with an increase of about $10 \%$ with respect to standard SLC representation and of about $8 \%$ when compared to PCA images.

\section{B. Flood mapping}

In this Section, the suitability of the proposed DL- $1 \alpha$ products for flood mapping is explored. The study area is the
Albufera natural reserve (Valencia, Spain). Here, business and environmental protection coexist [23]. In fact, the Albufera is one of the greatest Spanish rice production area, as well as an important biodiversity basin protected by the European Union under the aegis of the Natura 2000 Programme.

Rice cultivations need to be periodically flooded in order to ensure the best yield according with the plant life-cycle. However, in this case, flooding periods must be also ruled according with the needs of the species living in the reserve. For this reason, the area is subject to a continuous conflict between rice producers and park rangers willing to favor the business rather than the nature preservation.

In the following, the flooded areas have been mapped using the proposed DL- $1 \alpha$ products. A sample relevant with the case study is shown in Fig. 5a. The reference image (blue band) has been acquired in October 2017, thus after the rice harvesting, when the dominant land cover is bare soil. The test band (green channel) is an image acquired on 6 January 2017 in presence of flood. As explained in [2] this composition allows for rendering a temporary water coverage in blue color.

In Fig. 5b, a NDWI map [24] retrieved from a multispectral (MS) Landsat-8 acquisition made on 12 January 2017 is shown. The product resolution is 30 meter. Therefore, in order to have images fully comparable, the resolution of the SAR product has been degraded from 10 to 30 meter as well.

The reader should easily understand that, concerning the mapping of the flooded area, these two images qualitatively bring the same information. In order to extract quantitative information, standard thresholding is applied to the NDWI map and semantic clustering to the SAR product to extract pixels rendered in blue in the RGB map (see [25] for details).

NDWI-derived mask are assumed as the ground truth. The comparison with the SAR-derived map shows an overall 


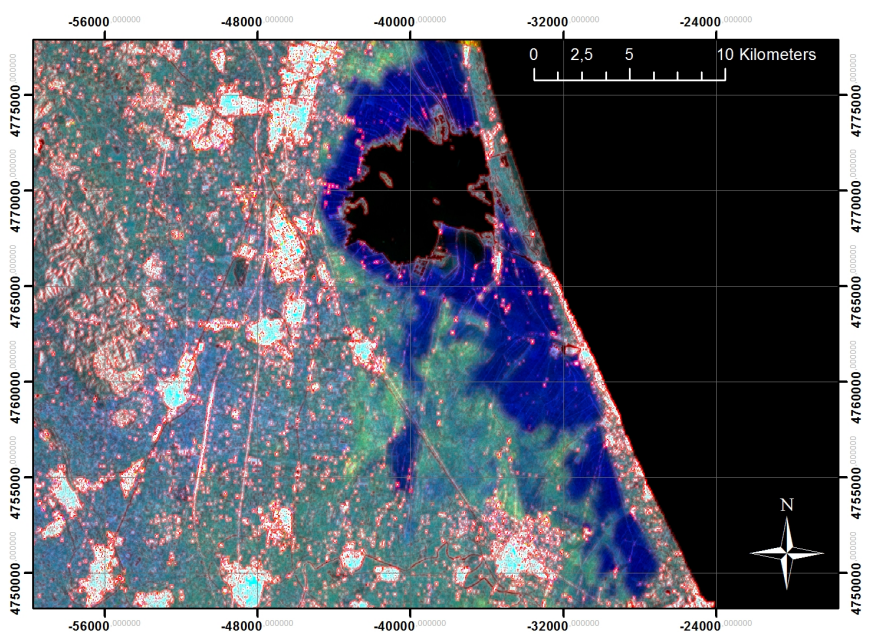

(a)

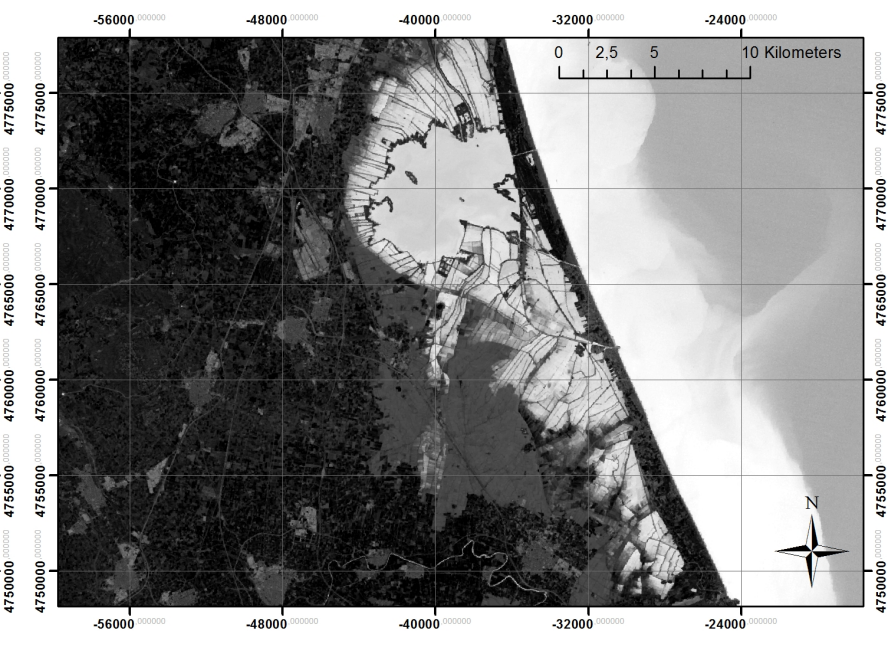

(b)

Fig. 5: Suitability of DL- $1 \alpha$ products with flood mapping applications, the Albufera (Spain) case study. (a) DL-1 $\alpha$ product (test image: 06/01/2017). (b) NDWI map derived from a Landsat-8 image acquired on 12/01/2017.

accuracy of $88.8 \%$ and a false alarm rate of $2.75 \%$. Taking into account that the two images have not been acquired exactly on the same day (there is about one week of time span), this result is in line with expectations. In fact, the effectiveness of standard L-1 $\alpha$ images with this application has been already demonstrated (see as an example [2] and [26]). Due to the equivalence of DL-1 $\alpha$ products, reliable results were expected as well. The advantage they introduce is in the possibility of building temporal series of RGB products by handling input images (i.e. the GRD products) less demanding from computational and storage viewpoints.

An example of the usage of DL- $1 \alpha$ products in the perspective of time series is depicted in Fig. 6 . All the products share the same reference image, acquired on October 2017. Test images have been acquired (from left to right and up to down) on 19 March 2017, 18 May 2017, 11 June 2017, 15 September 2017, 14 November 2017, and 20 December 2017. A quantitative comparison between the SAR derived flood map and the MS-derived flood map (retrieved via trialand error thresholding of relevant NDWI maps and assumed as reference) is provided in TABLE II for the dates in which both acquisitions are available (with a cloud free MS) with a temporal baseline of a few days. Due to the negligible role of the interferometric coherence in this application, the performance of L- $1 \alpha$ and DL- $1 \alpha$ images are the same. These values confirm the reliability of the information retrievable from RGB SAR, and that this approach allows for an effective continuous monitoring of the area, thus helping in the control of the correct timing of floods for the best management of the natural park resources.

\section{Urban area monitoring}

In this section, another classic application of SAR remote sensing, i.e. urban area monitoring, is addressed. In particular, the usage of the proposed DL- $1 \alpha$ products for monitoring
TABLE II: Quantitative comparison between SAR-derived and MS-derived flood maps.

\begin{tabular}{cccc}
\hline \hline Date SAR & Accuracy (\%) & False alarms (\%) & Date MS \\
\hline \hline $06 / 01 / 2017$ & 88.8 & 2.75 & $12 / 01 / 2017$ \\
$19 / 03 / 2017$ & 93.2 & 0.93 & $17 / 03 / 2017$ \\
$18 / 05 / 2017$ & 79.2 & 10.5 & $20 / 05 / 2017$ \\
$14 / 11 / 2017$ & 89.5 & 2.05 & $12 / 11 / 2017$ \\
\hline \hline
\end{tabular}

the growth of urban areas using long time series will be investigated.

The selected study area is the city of Chennai (India), which has undergone an enormous growth in the last twenty years. However, exploring the time-evolution of a city implies to deal with data acquired by different sensors, if and where possible. ESA's policy with regard to the Sentinel-1 mission is to ensure continuity with the previous Agency SAR sensors made exception for an obvious improvement in the technology available for the system design. This has made the combination of Sentinel-1 images with those acquired by the past ESA missions, namely ERS-1 (1992-2000), ERS-2 (1995-2011), and Envisat (2002-2012) possible, and it is here implemented according to the schema discussed in Section III

The result of this approach is depicted in Fig. 7. The reference image (associated with the blue band) is the oldest one acquired on the study area by the ERS-1 sensor in May 1992. Test images have been acquired (from left to right and up to down) in August 1992 and 1997 by ERS-1, 2003 and 2010 by Envisat, and 2015 and 2017 by Sentinel-1. The first RGB frame (involving two images acquired with short temporal baseline) represents the time zero. At this time, the urban area is mainly segregated near the coastline. Starting from 1995, it is possible to understand intuitively the spreading of the city towards the inland. At pixel level, the construction of new buildings causes an abrupt increase of the backscattering of the test image, as well as of the data range. Therefore, new 


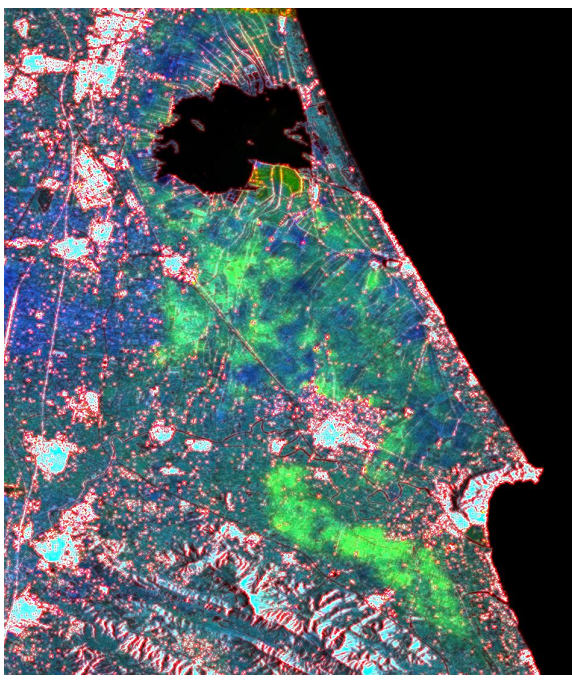

(a)

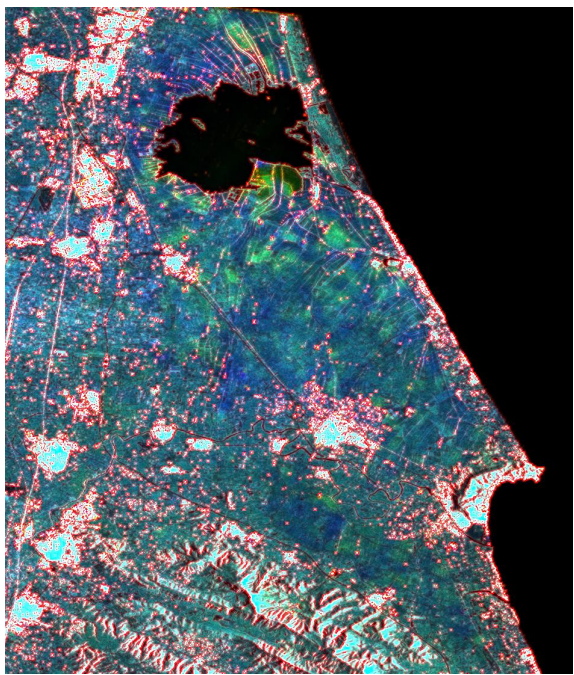

(d)

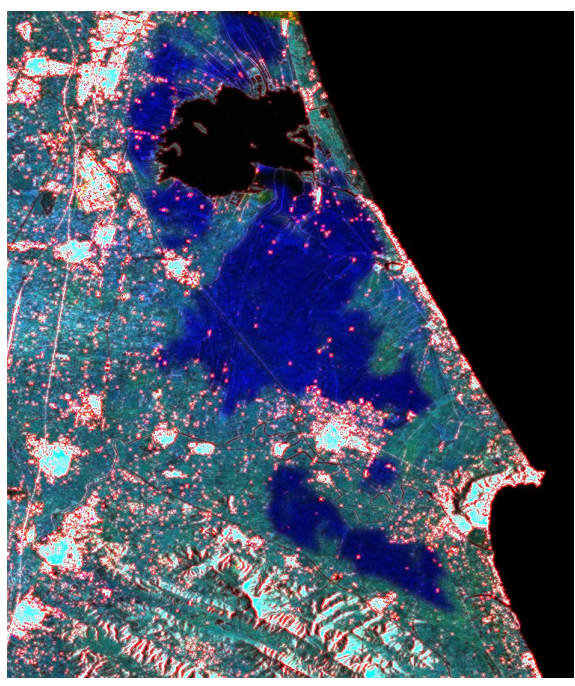

(b)

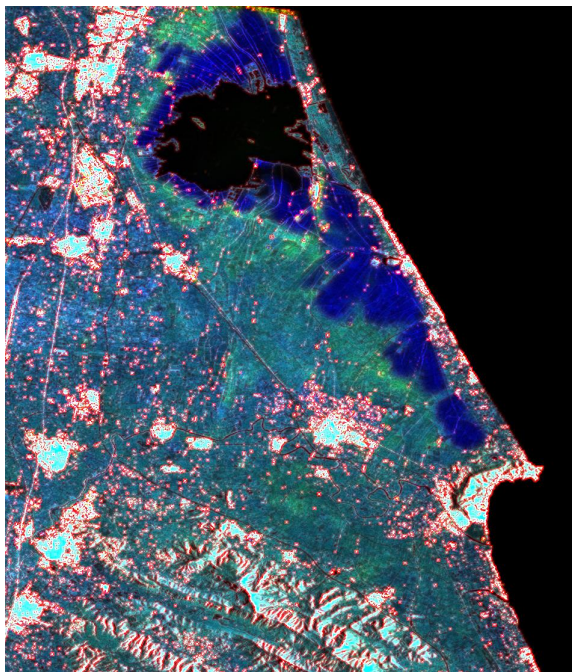

(e)

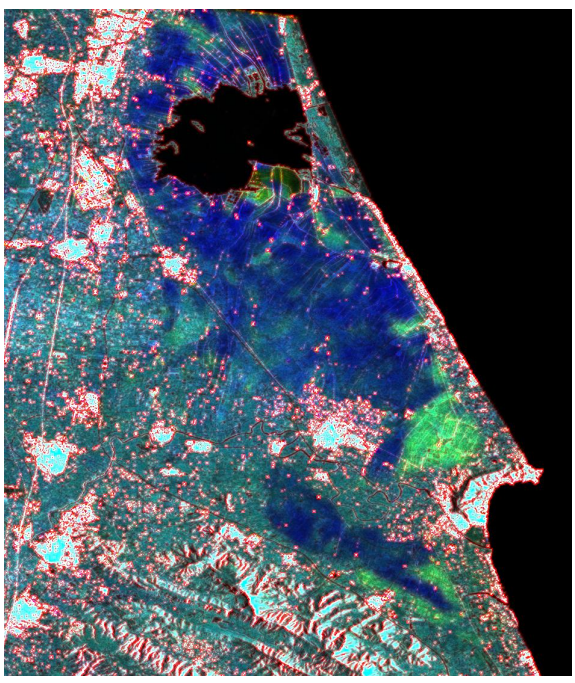

(c)

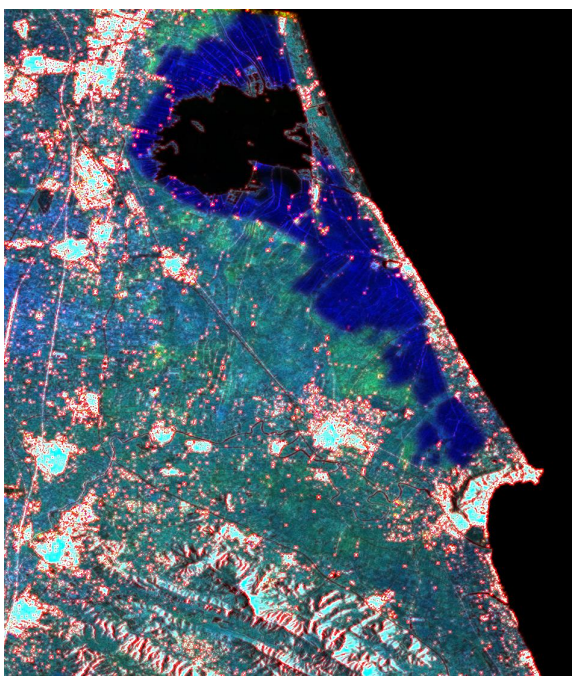

(f)

Fig. 6: Time series of DL- $1 \alpha$ products for continuous flood monitoring. Reference image acquired on October 2017. Test images acquired (from left to right and up to down) on 19 March 2017, 18 May 2017, 11 June 2017, 15 September 2017, 14 November 2017, and 20 December 2017.

built-up areas are expected to be depicted in yellow, and this is confirmed looking at Fig. 7 in which it can be appreciated that the built-up area has nearly doubled in 2017 with respect to 1992 . This is also confirmed by population data according to which the inhabitants increased from about 3.8 million in 1991 to about 7.1 million in 2011.

However, as previously discussed, highly dense urban area cause a different response of the data range texture. There, the lack of shadowed pixels makes this measure low, thus causing new built-up areas to be rendered in almost pure green color. To clarify this, let us examine the graphics reported in Fig. 8 . still concerning the city of Chennai. The color composite has been obtained by fusing two Sentinel-1 GRD products acquired in May 2015 and December 2017.

In the bottom part of the figure, a low-density built-up case is highlighted. A yellow ring, coming from the phenomenology above illustrated, is visible on the SAR image. The feature corresponds to a new isolated construction, as the Google Earth database shows. This can be appreciated by analyzing the two patches placed near the letter $A$ in the graphics. In fact, it is possible to observe that bare soil land cover of early 2015 is replaced by the same (under construction) ring visible in the SAR image in August 2017.

Slightly upper in Fig. 8, a high-density built-up case is reported. Here, a vegetated land cover is replaced by several industrial buildings creating a continuous texture causing the response of the DL- $1 \alpha$ product being represented by pure green color.

Finally, the performance of D-L1 $\alpha$ versus that of $\mathrm{L}-1 \alpha$ (assumed as reference) is quantitatively assessed by extracting from both products the urban area using semantic clustering as described in [25]. Three RGB products obtained from Sentinel-1 images relevant to years 2015, 2016, and 2017 have been tested. The results are summarized in TABLE III] They 

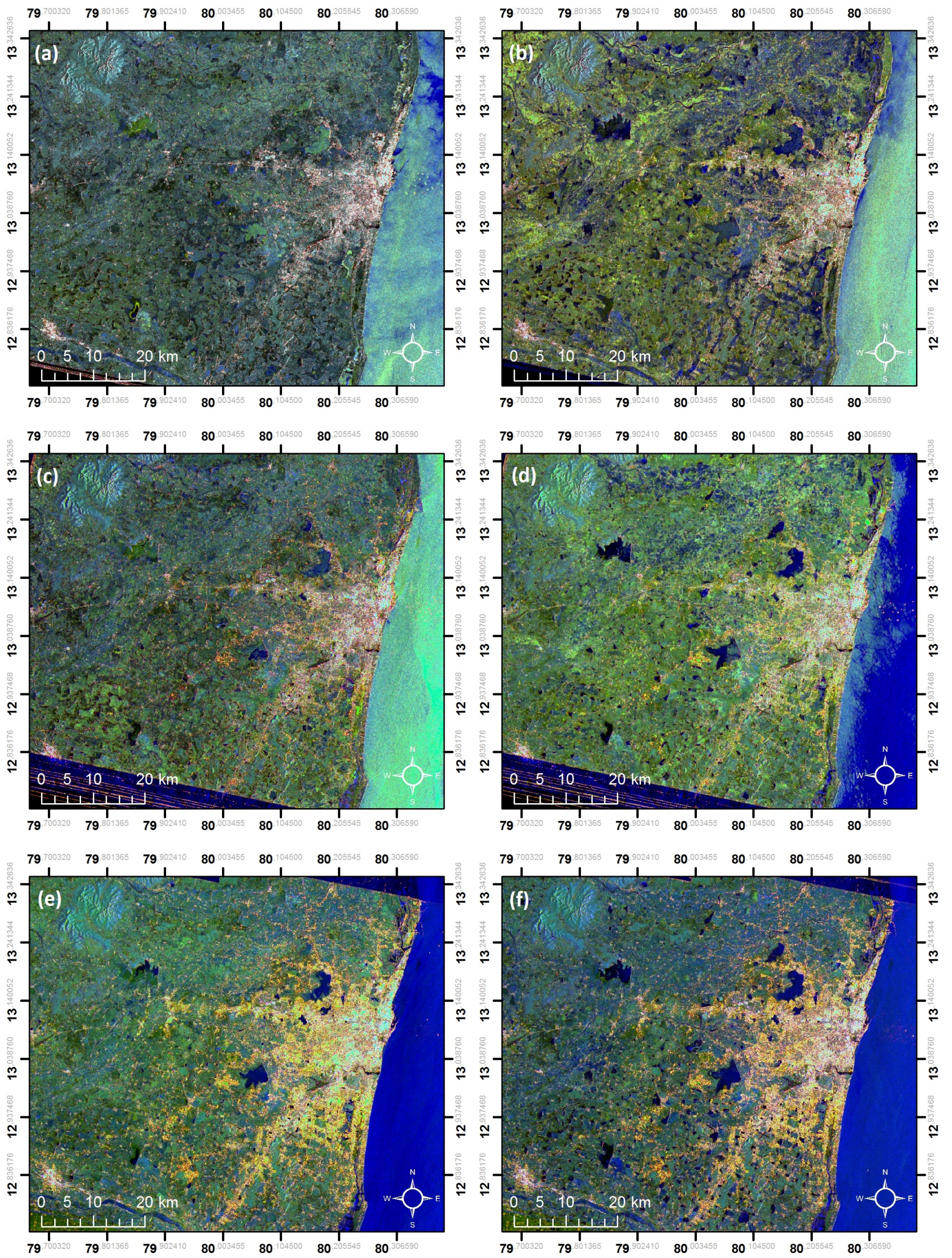

Fig. 7: Evolution of the Chennai urban area catched by a time series of DL- $1 \alpha$ products. Reference image acquired by ERS-1 sensor on May 1992. Test images acquired (from left to right and up to down) on August 1992 and 1997 by ERS-1, 2003 and 2010 by Envisat, and 2015 and 2017 by Sentinel-1 


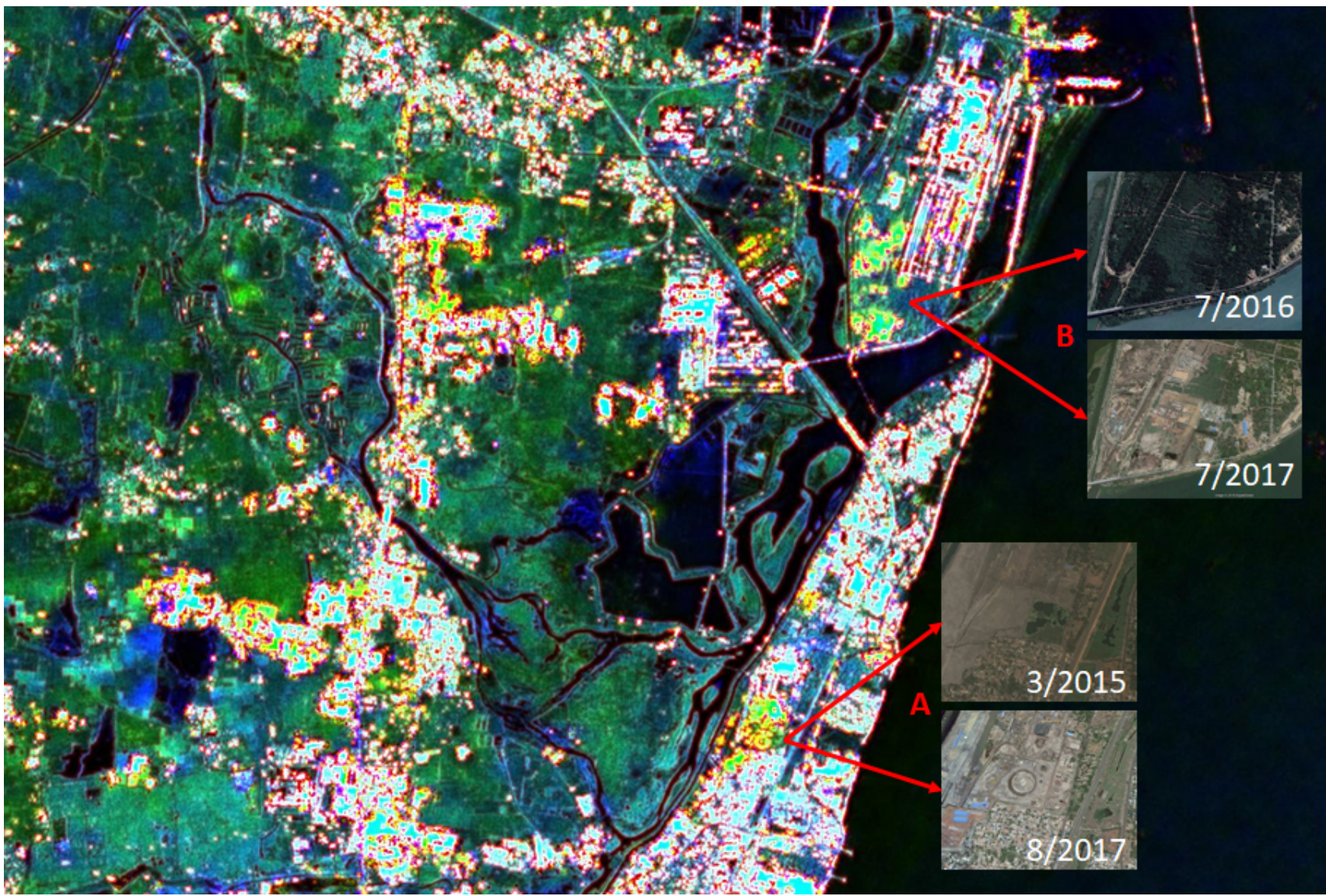

Fig. 8: Behavior of the proposed DL- $1 \alpha$ products with the new built-up area class. Its density determines the response of the data range texture making it high for low-density areas (thus displayed in yellow) and low for high-density areas (consequently rendered in almost pure green).

confirm that the information extracted from the two classes of products is fully comparable.

TABLE III: Urban area extraction using DL- $1 \alpha$ images, comparison with data derived from L- $1 \alpha$ products.

\begin{tabular}{ccc}
\hline \hline Year & Accuracy (\%) & False alarms (\%) \\
\hline \hline 2015 & 96.6 & 2.54 \\
2016 & 94.6 & 3.11 \\
2017 & 96.7 & 2.28 \\
\hline \hline
\end{tabular}

\section{Conclusions}

Since 2014, the Sentinel-1 mission has been providing high quality synthetic aperture radar images with high temporal resolution free of charge for both commercial and scientific users. This policy is boosting the downstream market in which easy-to-use products and simple processing chains for information extraction are required for setting-up operational services.

Among the products delivered by the Sentinel-1 mission, the ground range detected class is raising users' interests due to their availability on cloud platforms like the Google Earth Engine and the reduced computational load with respect to the corresponding for single look complex products. In this work, a new class of multitemporal products is introduced. Differently from the recently introduced RGB Level- $1 \alpha$ product, they allow for building temporal color composite without the need to exploit the phase information. The purpose is to provide a new class of products, application and user-oriented, which can replace the use of complex Level- $1 \alpha$ products when adequate computational power is not available and for all the applications in which the phase information is not a strict requirement.

The proposed processing chain was largely inspired by that bringing to the definition of the Level- $1 \alpha$ products. However, significant innovation is introduced to substitute the phase information with something able to convey comparable information, especially concerning the reconstruction of the built-up feature. This was identified in a texture measure, i.e. the data range. The proposed methodology is also suitable for multisensor data combination allowing for the combination of images acquired by different, but radiometrically and spatially comparable, platforms. (i.e. Sentinel-1, ERS-1/2, and Envisat), thus introducing new possibilities for the exploitation of archive images.

The effectiveness of the proposed methodology has been discussed in an applicative environment. Operative contexts exploiting the proposed Detected Level- $1 \alpha$ products have been explored relatively to image photo-interpretation, flood 
mapping and urban area monitoring.

In the first case, a perceptive test assessed that the use of such color composition allows for the improvement of the interpretability of several classes with respect to standard SAR representations.

Flood mapping has been addressed concerning the monitoring of the Albufera natural reserve. Urban area monitoring, instead, was applied in relation with the long term mapping of the evolution of the Chennai town. To this end, images acquired by different sensors have been successfully combined to identify new built-up areas. In both the applications, the use of the proposed products brought to results comparable with the state of the art in shorter times and at a lower computational load.

\section{ACKNOWLEDGMENTS}

The authors thank the European Space Agency for providing free of charge the archive ERS and Envisat images used in this study under the aegis of the project 41292 "Long term satellite observations for urban areas and water resources monitoring."

\section{REFERENCES}

[1] Z. Malenovský, H. Rott, J. Cihlar, M. E. Schaepman, G. GarcíaSantos, R. Fernandes, and M. Berger, "Sentinels for science: Potential of Sentinel-1, -2, and -3 missions for scientific observations of ocean, cryosphere, and land," Remote Sens. Environ., vol. 120, pp. 91-101, 2012.

[2] D. Amitrano, G. Di Martino, A. Iodice, D. Riccio, and G. Ruello, "A New Framework for SAR Multitemporal Data RGB Representation: Rationale and Products," IEEE Trans. Geosci. Remote Sens., vol. 53, no. 1, pp. 117-133, 2015.

[3] D. Amitrano, F. Cecinati, G. Di Martino, A. Iodice, P.-P. Mathieu, D. Riccio, and G. Ruello, "Multitemporal Level-1 $\beta$ Products: Definitions, Interpretation, and Applications," IEEE Trans. Geosci. Remote Sens., vol. 54, no. 11, pp. 6545-6562, 2016.

[4] E. I. Alves, A. I. A. S. S. Andrade, and D. A. Vaz, "A Better View Over Titan Drainage Networks Through RGB Fusion of Cassini SAR Images," IEEE Geosci. Remote Sens. Lett., vol. 15, pp. 414-418, 2018.

[5] S. G. Dellepiane and E. Angiati, "A New Method for CrossNormalization and Multitemporal Visualization of SAR Images for the Detection of Flooded Areas," IEEE Trans. Geosci. Remote Sens., vol. 50, no. 7, pp. 2765-2779, 2012.

[6] D. Amitrano, V. Belfiore, F. Cecinati, G. Di Martino, A. Iodice, P. P. Mathieu, S. Medagli, D. Poreh, D. Riccio, and G. Ruello, "Urban Areas Enhancement in Multitemporal SAR RGB Images Using Adaptive Coherence Window and Texture Information," IEEE J. Sel. Topics Appl. Earth Observ., vol. 9, no. 8, pp. 3740-3752, 2016.

[7] C.-Y. Chiang, K.-S. Chen, C.-Y. Chu, Y.-L. Chang, and K.-C. Fan, "Color enhancement for four-component decomposed polarimetric sar image based on a cie-lab encoding," Remote Sensing, vol. 10, no. 4 2018.

[8] D. Amitrano, G. Di Martino, A. Iodice, D. Riccio, and G. Ruello, "Unsupervised Rapid Flood Mapping Using Sentinel-1 GRD SAR Images," IEEE Trans. Geosci. Remote Sens., In press.

[9] G. Donchyts, F. Baart, H. Winsemius, N. Gorelick, J. Kwadijk, and N. van de Giesen, "Earth's surface water change over the past 30 years," Nature Climate Change, vol. 6, no. 9, pp. 810-813, 2016.

[10] N. Gorelick, M. Hancher, M. Dixon, S. Ilyushchenko, D. Thau, and R. Moore, "Google Earth Engine: Planetary-scale geospatial analysis for everyone," Remote Sensing of Environment, In press.

[11] G. Donchyts, J. Schellekens, H. Winsemius, E. Eisemann, and N. van de Giesen, "A 30 m Resolution Surface Water Mask Including Estimation of Positional and Thematic Differences Using Landsat 8, SRTM and OpenStreetMap: A Case Study in the Murray-Darling Basin, Australia," Remote Sens., vol. 8, no. 5, 2016.

[12] Y. Yamaguchi, T. Moriyama, M. Ishido, and H. Yamada, "Fourcomponent scattering model for polarimetric sar image decomposition," IEEE Trans. Geosci. Remote Sens., vol. 43, no. 8, pp. 1699-1706, 2005.
[13] J.-S. Lee and E. Pottier, Polarimetric Radar Imaging: From Basics to Applications. Boca Raton, FL: CRC Press, 2009.

[14] A. Mouratidis and F. Sarti, "Flash-flood monitoring and damage assessment with SAR data: Issues and future challenges for Earth observation from space sustained by case studies from the Balkans and Eastern Europe," in Earth Observation of Global Changes, ser. Lecture Notes in Geoinformation and Cartography, J. M. Krisp, L. Mend, R. Pail, and U. Stilla, Eds. Berlin Heidelberg: Springer, 2013.

[15] F. Siegert and A. A. Hoffmann, "The 1998 Forest Fires in East Kalimantan (Indonesia): A Quantitative Evaluation Using High Resolution, Multitemporal ERS-2 SAR Images and NOAA-AVHRR Hotspot Data," Remote Sens. Environ., vol. 72, no. 1, pp. 64-77, 1998.

[16] P. Nakmuenwai, F. Yamazaki, , and W. Liu, "Multi-temporal correlation method for damage assessment of buildings from high- resolution sar images of the 2013 typhoon haiyan," Journal of Disaster Research, vol. 11, no. 3, pp. 577-592, 2016.

[17] M. Schmitt, L. H. Hughes, M. Korner, and X. X. Zhu, "Colorizing Sentinel-1 SAR Images Using a Variational Autoencoder Conditioned on Sentinel-2 imagery," in The International Archives of the Photogrammetry, Remote Sensing and Spatial Information Sciences, Volume XLII-2, 2018.

[18] N. P. Jacobson and M. R. Gupta, "Design Goals and Solutions for Display of Hyperspectral Images," IEEE Trans. Geosci. Remote Sens., vol. 43, no. 11, pp. 2684-2692, 2005.

[19] M. A. Clement, C. G. Kilsby, and P. Moore, "Multi-temporal synthetic aperture radar flood mapping using change detection," Journal of Flood Risk Management, pp. 1-17, 2017.

[20] G. F. De Grandi, M. Leysen, J.-S. Lee, and D. Schuler, "Radar reflectivity estimation using multiple SAR scenes of the same target: technique and applications," in IEEE Int. Geosci. Remote Sens. Symp., 1997, pp. 1047-1050.

[21] R. F. Hanssen, Radar Interferometry - Data Interpretation and Error Analysis. Dordrecht: Kluwer Academic Publishers, 2001.

[22] R. Touzi and A. Lopes, "Statistics of the Stokes Parameters and of the Complex Coherence Parameters in One-Look and Multilook Speckle Fields," IEEE Trans. Geosci. Remote Sens., vol. 34, no. 2, pp. 519-531, 1996.

[23] J. M. Soria, "Past, present and future of la Albufera of Valencia Natural Park," Limnetica, vol. 25, no. 1-2, pp. 135-142, 2006.

[24] S. K. McFeeters, "The use of the Normalized Difference Water Index (NDWI) in the delineation of open water features," Int. J. Remote Sens., vol. 17, no. 7, pp. 1425-1432, 1996.

[25] D. Amitrano, F. Cecinati, G. Di Martino, A. Iodice, P.-P. Mathieu D. Riccio, and G. Ruello, "Feature Extraction From Multitemporal SAR Images Using Selforganizing Map Clustering and Object-Based Image Analysis," IEEE J. Sel. Topics Appl. Earth Observ., vol. 11, no. 5, pp. 1556-1570, 2018.

[26] D. Amitrano, G. Di Martino, A. Iodice, F. Mitidieri, M. N. Papa, D. Riccio, and G. Ruello, "Sentinel-1 for Monitoring Reservoirs: A Performance Analysis," Remote Sens., vol. 6, pp. 10676-10 693, 2014. 